\title{
Ursell operators in statistical physics I: Generalizing the Beth Uhlenbeck formula.
}

\author{
F. Laloë \\ Laboratoire Kastler Brossel *
}

March 22, 2018

\begin{abstract}
The Beth Uhlenbeck formula gives an exact (quantum) expression of the second virial correction to the equation of state of a dilute gas. We show how this result can be extended to arbitrary degeneracy. For this purpose we develop a formalism based on the use of Ursell operators, which contain no symmetrization in themselves (they can be used for both a system of identical particles or an auxiliary system of distinguishable particles). A concise expression generalizing the Beth Uhlenbeck formula is obtained, which is equally valid for bosons and fermions. The formalism is not essentially limited to dilute gases, but can also be applied to the study of denser systems, for instance those undergoing a Bose Einstein transition; this will be the subject of a forthcoming article.
\end{abstract}

\section{Introduction}

The Beth Uhlenbeck formula [1] [2] gives an exact expression of the second virial coefficient as a function of all collision phase shifts associated with the interaction potential. This is a remarkable result which, in a concise formula including no phenomenological constant, relates macroscopic and microscopic quantities: on one hand the density, the temperature, and the pressure of a gas, on the other the quantum phase shifts, which are exactly calculable from the Schrödinger equation. A closer inspection shows that the pressure correction is the sum of two contributions, one of pure statistics, and a second arising from the interactions; the former is actually nothing but the first order term of the expansion in $n \lambda^{3}$ of the ideal gas equation of state. It is therefore not surprising that the validity of the formula should require two conditions, that the gas be dilute with respect

*UA associée au CNRS no 18, laboratoire associé à l'Université Pierre et Marie Curie. 
to both degeneracy effects $\left(n \lambda^{3} \ll 1\right)$ and interaction effects as well $\left(n b^{3} \ll 1\right.$, where $b$ is the range of the interaction potential). A natural question is whether one can release the first of these conditions and study gaseous systems over a larger range, going continuously from the classical region where $n \lambda^{3} \ll 1$ to the quantum region where this quantity is comparable to one. One would just assume that the gas remains always dilute in terms of the interactions. As a matter of fact, most classical textbooks on quantum statistical mechanics include a study of gaseous systems that are degenerate and imperfect. The methods they use are based on various forms of perturbation theory; for instance they introduce an auxiliary pseudopotential which replaces the real interaction potential [3], or they make a direct substitution of a collision $T$ matrix for this potential [4]. This well known approaches are, nevertheless, different from that of Beth and Uhlenbeck whose calculation is exact (of course they limit the calculation to the first density correction to the equation of state, but this is precisely the definition of the second virial correction). In essence, the cluster expansion method they use is a method providing directly density expansions, as opposed to interaction expansions, which require at least partial resummations of diagrams in powers of the interaction potential [5] to reconstruct the $T$ matrix from $V$ and to obtain density expansions. Actually, the exact treatment of the relative motion of two particles is explicit in the Beth Uhlenbeck formula, which contains a thermal exponential of the two particle hamiltonian ensuring that thermal equilibrium has been reached, in other words that the binary correlations between the particles are treated properly. Technically, a characteristic of this approach is that no expression which becomes infinite for repulsive hard cores is ever written, even at some intermediate stage. It therefore seems to be an attractive possibility to try and extend the method to degenerate systems, if only to compare the result of both methods, interaction and density expansions. This is the subject of the present article; we show that concise and exact formulas can indeed be written that provide a natural generalization of the Beth Uhlenbeck formula, without requiring complicated algebra.

For this purpose, we will make use of a generalization of the quantum Ursell cluster functions introduced by Kahn and Uhlenbeck [6]: Ursell operators which intrinsically do not contain statistics (as opposed to the usual quantum Ursell functions [8] [9], which are already symmetrized). The action of the operators is defined, not only in the space of symmetric or antisymmetric states of the real system, but also in the larger space of an auxiliary system of distinguishable particles. If the system is dilute in terms of the interactions but not of statistics, this makes it possible to limit the calculation to low order Ursell operators while, with the usual (fully symmetrized) Ursell functions, one would need to include

\footnotetext{
${ }^{1}$ The initial introduction of the Ursell functions was made by him within classical statistical mechanics [7], and generalized ten years later to quantum mechanics by Kahn and Uhlenbeck.
} 
higher and higher orders with increasing degeneracyf. Of course, this method implies that we have to give up well-known advantages of the formalism of second quantization, but this is the price to pay for the treatment of interactions and statistics in completely independent steps. It also means that an explicit symmetrization of the wave functions becomes indispensable at some point and, moreover, that no approximation whatsoever can be made at this step: otherwise the possibility of treating strongly degenerate systems would be lost. Fortunately it turns out that the symmetrization operation can indeed be performed exactly. This is done by introducing simple products of operators, corresponding to exchange cycles, or more generally simple functions of operators (fractions) that correspond to summations over the size of these cycles up to infinity. Physically, this emphasizes the relation which exists between the properties of a system, its pressure for instance, and the size of exchange cycles 10 that take place in it.

Another feature of the method is that it naturally provides expressions for the one and two body density operators, which is of course convenient if one is interested in a detailed study of correlations. The formalism is useful beyond the evaluation of virial corrections only; this is why we develop it in what follows beyond what is strictly necessary to generalize the Beth Uhlenbeck formula. A preliminary report on this work has already been given in [10]; applications to denser systems will be the object of a forthcoming article 11].

\section{Ursell operators}

In the canonical ensemble, the partition function $Z_{N}$ of a system of indistinguishable particles is given by a trace inside the space of symmetrical (or antisymmetrical) states. Nevertheless, with the help of the projectors $S$ and $A$ onto these (sub)spaces, the trace can be extended to a larger space, which is associated with the same number of distinguishable particles:

$$
Z_{N}=\operatorname{Tr}\left\{K_{N}^{S}\right\}
$$

In this equation, $S$ applies for bosons while $A$ applies for fermions, and the operators $K_{N}$ are defined by:

$$
\begin{aligned}
K_{1} & =\exp -\beta H_{0}(1) \\
K_{2} & =\exp -\beta\left[H_{0}(1)+H_{0}(2)+V_{12}\right] \\
\cdots & \cdots \\
K_{N} & =\exp -\beta\left[\sum_{i=1}^{N} H_{0}(i)+\sum_{i>j} V_{i j}\right]
\end{aligned}
$$

\footnotetext{
${ }^{2}$ As discussed in [10], when a Bose Einstein condensation takes place, the Ursell operators have no singular variation, which is another way to see that they are not sensitive to degeneracy by themselves.
} 
with usual notation: $H_{0}(1)$ is the one particle hamiltonian including kinetic energy and an external potential (if there is any in the problem), $V_{i j}$ is the interaction potential between particles with labels $i$ and $j$. We now use cluster techniques to expand operators exactly in the same way as one usually does for functions. The Ursell operators $U_{l}$ are therefore defined according to:

$$
\begin{aligned}
U_{1}= & K_{1} \\
U_{2}(1,2)= & K_{2}(1,2)-K_{1}(1) K_{1}(2) \\
U_{3}(1,2,3)= & K_{3}(1,2,3)-K_{2}(1,2) K_{1}(3)-K_{2}(2,3) K_{1}(1) \\
& -K_{2}(3,1) K_{1}(2)+2 K_{1}(1) K_{2}(2) K_{1}(3)
\end{aligned}
$$

etc. $(l=1,2, . . N)$. Conversely, in terms of the Ursell operators, the $N$ particle operator $K_{N}$ can be written in the form:

$$
K_{N}=\sum_{\left\{m_{l}^{\prime}\right\}} \sum_{\left\{D^{\prime}\right\}} \underbrace{U_{1}(.) U_{1}(.) \ldots U_{1}(.)}_{m_{1}^{\prime} \text { factors }} \times \underbrace{U_{2}(., .) U_{2}(., .) \ldots U_{2}(., .)}_{m_{2}^{\prime} \text { factors }} \times U_{3}(., ., .) \ldots
$$

where the first summation is made on all possible ways to decompose the number of particles as:

$$
N=\sum_{l} l m_{l}^{\prime}
$$

The second summation correspond to all non-equivalent? ways to distribute the $N$ particles into the variables of the Ursell operators, symbolized by dots in (4). It is convenient to simplify the two summations into one:

$$
\sum_{\left\{m_{i}^{\prime}\right\}} \sum_{\left\{D^{\prime}\right\}} \Longrightarrow \sum_{\{U\}}
$$

which is meant over all non-equivalent ways to distribute the particles into various sequences of $U$ 's. There is little difference between our definitions and those of section 4.2 of [8] or [9]: we use operators instead of symmetrized functions and, more importantly, the action of these operators are defined, not only within the state space that is appropriate for bosons or fermions, but also in the larger space obtained by tensor product that occurs for distinguishable particles. Hence the need for an explicit inclusion of $S$ or $A$ in (1).

We now decompose these operators into a sum of permutations $P_{\alpha}$ that, in

\footnotetext{
${ }^{3}$ For instance $U_{2}(1,2) U_{3}(3,4,5)$ and $U_{2}(2,1) U_{3}(4,3,5)$ correspond to equivalent distributions of 5 particles, since the very definition of the Ursell operators implies that the order inside each $U_{k}$ is irrelevant.
} 
turn, we decompose into independent cycles $C$ of particlest:

$$
\begin{gathered}
S \\
A
\end{gathered}=\frac{1}{N !} \sum_{\left\{m_{i}\right\}} \sum_{\{D\}} \underbrace{C_{1}(.) C_{1}(.) C_{1}(.)}_{m_{1} \text { factors }} \times \eta^{m_{2}} \underbrace{C_{2}(., .) C_{2}(., .) C_{2}(., .)}_{m_{2} \text { factors }} \times C_{3}(., ., .) \ldots
$$

where the first summation is similar to that of (4), while the second corresponds to all non-equivalent ways to distribute numbers ranging from one to $N$ into the variables of the various $C$ 's. In this equation, the operator $S$ applies for bosons with $\eta=+1$, while $A$ with $\eta=-1$ applies for fermions. We also simplify the notation into:

$$
\sum_{\left\{m_{i}\right\}} \sum_{\{D\}} \Longrightarrow \sum_{\left\{P_{\alpha}\right\}}
$$

We can now insert (四) and (17) into (四) and obtain, within a double summation, numbers that are traces calculated in the space of distinguishable particles, i.e. in the ordinary tensor product of $N$ single particle state spaces. Inside most of the terms of the summation, factorization into traces taken inside smaller subspaces occur. For instance, if the term in question contains particle number $i$ contained at the same time in a $U_{1}$ operator as well as in a $C_{1}$, the contribution of that particle completely separates by introducing the simple number $\operatorname{Tr}\left\{U_{1}\right\}$. Or, if $n$ particles are all in the same $U_{n}$ but all in separate $C_{1}$ 's, this group of particles contributes by a factor $\operatorname{Tr}_{1 . . n}\left\{U_{n}\right\}$; if they are all in different $U_{1}$ 's but also contained in one single large cycle $C_{n}$, their contribution also factorizes separately. More generally, in each term of the double summation, particles group into clusters (U-C clusters), which associate together all particles that are linked either by cycles $C_{l}$ (with $l>1$ ) or by Ursell operators $U_{l^{\prime}}\left(\right.$ with $l^{\prime}>1$ ). The general term is therefore the product of the contributions of all the clusters that it contains and one can write:

$$
Z_{N}=\frac{1}{N !} \sum_{\left\{P_{\alpha}\right\}} \sum_{\{U\}} \prod_{\text {clusters }} \Gamma_{\text {cluster }}(i, j, k, . .)
$$

where $(i, j, k, .$.$) is the index number of particles contained inside the cluster; the$ number of clusters into which each term of the double summation is factorized depends, in general, on this particular term.

\footnotetext{
${ }^{4}$ We use the same notation as in ref. [10]: $C_{k}(i, j, k, .$.$) denotes a cycle where particle i$ replaces particle $j$, particle $j$ replaces particle $k$, etc. This should not be confused with the notation $P(i, j, k, \ldots)$ for a general permutation (not necessarily a cycle) where particle $i$ replaces particle 1 , particle $j$ particle 2, particle $k$ particle 3, etc. Any $P$ can be decomposed into a product of $C^{\prime}$ 's; for instance, $P(2,1,3)=C_{2}(1,2) C_{1}(3)$.

${ }^{5}$ Inside every cycle, a circular permutation of the variables has no effect and therefore does not affect the permutation $P_{\alpha}$.

${ }^{6}$ There are therefore two explicitly distinct origins to the clustering of particles in this point of view: belonging to the same $U_{l^{\prime}}\left(\right.$ with $l^{\prime}>1$ ) or to the same cycle $C_{l}$ (with $l>1$ ). This is distinct from usual cluster theories where, either only interactions introduce clustering (as in classical statistical mechanics), or the two origins are not explicitely distinguished (as in usual quantum cluster theory).
} 


\section{Diagrams}

Clusters differing only by the numbering of particles that they contain give the same contribution. It is therefore useful to reason in terms of diagrams (U-C diagrams), which emphasize the way particles are connected through exchange cycles and Ursell operators, rather than their numbering. For instance, the first diagram will correspond to one particle in a $U_{1}$ and in a $C_{1}$ (whatever the numbering of the particle is), and contribute the value $\operatorname{Tr}\left\{U_{1}\right\}$ as mentioned above; another diagram will introduce the value $\operatorname{Tr}_{1,2}\left\{U_{2}\right\}$, etc.; more generally, the value of any diagram containing $n_{\text {diag }}$. particles can be written as a trace over the variables of $n_{\text {diag. }}$ particles numbered arbitrarily:

$$
\begin{aligned}
\Gamma_{\text {diag. }}= & \eta^{p_{2}+p_{4}+. .} \operatorname{Tr}_{1,2, . . n_{\text {diag. }}}\left\{U_{1}(1) \times\right. \\
& \left.\times . . U_{1}\left(r_{1}\right) U_{2}\left(r_{1}+1, r_{1}+2\right) \ldots C_{1}(i) \ldots C_{1}(j) C_{2}(l, s) \ldots\right\}
\end{aligned}
$$

Here $p_{k}$ is the number of cycles $\square$ of length $k$; the factor $\eta^{p_{2}+p_{4}+. .}$ arises from the factors $\eta$ 's in (17) and corresponds to the contribution of the parity of the permutations contained in the diagram to the total permutation of the $\mathrm{N}$ particles.

Now, inside each term of the multiple summation, a given diagram $\Gamma_{\text {diag }}$ may occur several times; we then note $m_{\text {diag }}$. the number of times it is repeated, and we get:

$$
Z_{N}=\frac{1}{N !} \sum_{\left\{P_{\alpha}\right\}} \sum_{\{U\}} \prod_{\text {diag. }}\left[\Gamma_{\text {diag. }}\right]^{m_{\text {diag }} .}
$$

with the obvious relation:

$$
N=\sum_{\text {diag. }} m_{\text {diag. }} \times n_{\text {diag. }}
$$

Of course identical diagrams appear, not only in the same term of the double summation, but also in many different terms. Therefore, if $\left.\sum_{\left\{m_{\text {diag }}\right\}}\right\}$ symbolizes a summation over all possible ways to decompose $N$ according to (12), we can also write:

$$
Z_{N}=\frac{1}{N !} \sum_{\left\{m_{\text {diag. }}\right\}} c\left\{m_{\text {diag. }}\right\} \times \prod_{\text {diag. }}\left[\Gamma_{\text {diag. }}\right]^{m_{\text {diag }} .}
$$

where $c\left\{m_{\text {diag. }}\right\}$ is the numbers of terms in the double summation of (11) that correspond to this particular decomposition of $N$.

To evaluate this number, we have to specify more precisely how the U-C diagrams are constructed graphically. In every cluster, we represent the permutation cycles $C_{k}$ by horizontal lines containing $k$ boxes, or segments, which are available to numbered particles. When the corresponding particles are inside $U_{1}$ 's, we do not add anything to the diagram; when they are contained inside $U_{2}$ 's, we join

\footnotetext{
${ }^{7}$ One obviously has: $p_{1}+2 p_{2}+\ldots=n_{\text {diag. }}$.
} 
the corresponding segments by an additional double line, a triple line for $U_{3}$ 's, etc. For instance, if we consider the pure exchange cluster:

$$
\Gamma_{\text {cluster }}=\operatorname{Tr}_{1,2, . ., 7}\left\{U_{1}(1) U_{1}(2) . . U_{1}(7) C_{7}(1,2, \ldots .7)\right\}
$$

the corresponding diagram will be that of figure 1-a. This kind of linear diagram is the only possibility for an ideal gas; to generate a $\Gamma_{\text {cluster }}$ appearing in (9), it must receive a numbered particle in the first segment of the line, in the second the particle which is replaced by it under the effect of the permutation cycle, in the third the particle which is replaced by that in the second segment, etc. Equation (14) gives what we will call the "explicit value" of the contribution of the diagram; using simple transformations (see ref. [10] or section 5.1), one can obtain the simpler "reduced value" $\operatorname{Tr}_{1}\left\{\left[U_{1}(1)\right]^{7}\right\}$.

If we now start from a cluster which contains one single $U_{2}$ :

$$
\Gamma_{\text {cluster }}=\operatorname{Tr}_{1,2, \ldots . .9}\left\{U_{2}(1,2) U_{1}(3) U_{1}(4) U_{1}(5) . . U_{1}(9) C_{3}(1,3,4) C_{6}(2,5, \ldots .9)\right\}
$$

we obtain the diagram shown in figures 1-b; in the same way, the cluster:

$$
\Gamma_{\text {cluster }}=\operatorname{Tr}_{1,2, \ldots . .10}\left\{U_{2}(1,6) U_{1}(2) U_{1}(3) . . U_{1}(10) C_{10}(1,2,3, ., 6, . .10)\right\}
$$

leads to the diagram of fig. 1-c. These two kinds of diagrams turn out to be the only ones that are necessary to generalize the Beth Uhlenbeck formula; we give their reduced values in section 5.2. Fig. 1-d shows an example of a diagram containing one single $U_{3}$, and arising from the trace:

$$
\Gamma=\operatorname{Tr}_{1,2, \ldots ., 16}\left\{U_{3}(1,2,3) U_{1}(4) U_{1}(5) . . U_{1}(13) C_{3}(1,4,5) C_{8}(2,6 . .12) C_{5}(3, . .16)\right\}
$$

and figure 1-e gives another similar example. Clearly, the process can be generalized to associate a diagram to any cluster, however large and complex.

But it is not sufficient to construct diagrams, we must also choose explicit rules ensuring that every $\Gamma_{\text {cluster }}$ appearing in (9), where the particles are still numbered, will correspond to one single, well defined, diagram; for instance, for the cluster written in (15), we must decide whether the diagram will be that shown in figure 1-b or another where the lowest cycle ("base cycle") is that of length 6 instead of 3 . We will avoid this kind of ambiguity by choosing rules which fix, for every cluster associated with a given diagram, where exactly each numbered particle should fall into the diagram. These rules ensure that no double counting of clusters may occur; they also determine how the geometrical characteristics of the diagrams (the lengths of the successive cycles) can be varied, which in turn determines the way in which the summations over lengths will be made in a second step (next section). For our purpose in this article, which is limited

\footnotetext{
${ }^{8}$ There is some flexibility in this choice, and here we attempt to take the most convenient convention, but it is not necessarily the only possiblity.
} 
to a generalization of the Beth Uhlenbeck formula and to the diagrams of figure $1-\mathrm{a}, \mathrm{b}$ and $\mathrm{c}$, we do not need to study the most general case (this discussion is given in Appendix I); it will be sufficient to remember that the first particle in the lowest cycle ("base cycle") must be, among all particles contained in the $U_{l}$ of highest order $l$, that which has the lowest index number for the configuration to be correct.

The preceding rules also introduce the "counting factors" corresponding to the probability of obtaining a correct representation of a cluster by throwing randomly numbered particles into a diagram; the counting factors are used below to obtain the value of $c\left\{m_{\text {diag. }}\right\}$. Suppose for instance that we distribute $k$ numbered particles into a linear diagram of the kind shown in fig. 1-a in all possible ways; it is clear that there is a probability $1 / k$ that the first particles will have the lowest index number as required, which leads to the following counting factor $f_{\text {diag. }}$ for pure exchange cycles:

$$
f_{\text {diag. }}=\frac{1}{k}
$$

(in other words, if one puts numbered particles into a cycle $C_{k}(., ., ., .$.$) , one can$ obtain the same permutation $k$ different times). Similarly, it is easy to see that the counting factors of the diagrams of figures 1 - $\mathrm{b}$ and $\mathrm{c}$ are $1 / 2$, which corresponds to the probability of having the particle numbers contained inside the single $U_{2}$ in the correct order. The general value of counting factors is given in Appendix I; in many practical situations (when case (ii) of this Appendix does not occur) we can ignore the $g$ 's in formula (62) and use the simpler form:

$$
f_{\text {diag. }}=\left[p_{l_{M}} \times l_{M}\right]^{-1}
$$

where $l_{M}$ is the largest order of the Ursell operator contained in the diagram (one for fig. 1-a, two for figs. b and c, three for figs. 1-d and e) while $p_{l_{M}}$ is the number of these Ursell operators in this particular diagram (7 for fig. 1-a, one for all the other cases in this figure). Two other examples of diagrams for which this formula is valid are given in figure 2; as an illustration, they are explicitly calculated in Appendix II.

We are now in position to calculate $c\left\{m_{\text {diag. }}\right\}$. This number can be obtained by distributing the $N$ particles inside the sites of all the diagrams of a series defined by the $m$ 's, which can be done in $N$ ! different ways, and counting how many times the same term of the double summation of (11) is obtained. Since there are $m_{\text {diag }}$ ! ways to interchange the order of all U-C clusters arising from the same diagram, there is a first redundancy factor equal to $\prod_{\text {diag. }}\left(m_{\text {diag }} !\right)$ that comes in. Moreover, there is also only a proportion $\left(f_{\text {diag. }}\right)^{m_{\text {diag. }}}$ of the obtained configurations that is acceptable. Altogether, the net result is:

$$
c\left\{m_{\text {diag. }}\right\}=N ! \prod_{\text {diag. }} \frac{1}{m_{\text {diag. } .}}\left[f_{\text {diag. }}\right]^{m_{\text {diag. }} .}
$$




\section{Two summations}

We now take advantage of the fact that (20) contains factorials and, in a second step, that the value of $f_{\text {diag. }}$ depends on the topology of the diagrams, but not of the size of the chains of $U_{1}$ 's that it contains (except for pure exchange sycles). This allows us to group together series of terms in (13). The first summation is done classically by going to the grand canonical ensemble and defining the corresponding partition function by:

$$
Z_{\text {g.c. }}=\sum_{N} e^{\beta \mu N} Z_{N}
$$

Then a useful simplification occurs because the sums over the $m_{\text {diag. }}$ 's are now independent; moreover the factors $e^{\beta \mu N}$ can be reconstructed by multiplying every number $\Gamma_{\text {diag. }}$ by $e^{\beta \mu n_{\text {diag. }} \text {, so that: }}$

$$
Z_{\text {g.c. }}=\prod_{\text {diag. }} \exp \left[\exp \left(\beta \mu n_{\text {diag. }}\right) \times f_{\text {diag. }} \times \Gamma_{\text {diag. }}\right]
$$

We therefore obtain the grand potential (multiplied by $-\beta$ ) in the form:

$$
\log Z_{\text {g.c. }}=\sum_{\text {diag. }} e^{\beta \mu n_{\text {diag. }}} \times f_{\text {diag. }} \times \Gamma_{\text {diag. }}
$$

This is an exact formula, which gives the value of the pressure of the system (multiplied by its volume and divided by the temperature).

The second summation which can now be done consists in grouping together the contributions of all U-C diagrams that have the same "frame" (or "skeleton); in other words we sum the diagrams which have the same topology and differ only, inside the horizontal lines that represent the permutation sycles, by the lengths of the intermediate chains of $U_{1}$ 's that connect together the particles contained in the $U_{l}$ 's with $l>1$. Beside the fact that this operation turns out to be mathematically simple - it merely leads to the introduction of fractions of the operator $U_{1}$ as we will see in the next section (see also the discussion of $\S 2$ of ref. [10]) -, it is also indispensable from a physical point of view: we have to make a summation over all lengths of intermediate horizontal chains of $U_{1}$ 's in order to take into account an arbitrary degree of degeneracy. We call "classes" these groups of topologically equivalent U-C diagrams; classes may also be represented by diagrams ( $\Xi$-diagrams) which are, in a sense, simpler than the original diagrams since any indication of the length of the cycles has been removed. Examples are shown in figure 3 where dashed lines emphasize that a summation over cycle length is implied. We call $\Xi_{\text {class }}$ the contribution of a class:

$$
\Xi_{\text {class }}=\sum_{\text {diag. } \in \text { class }} e^{\beta \mu n_{\text {diag. }}} \times f_{\text {diag. }} \times \Gamma_{\text {diag. }}
$$


In terms of classes, (23) becomes:

$$
\log Z_{\text {g.c. }}=\sum_{\text {classes }} \Xi_{\text {class }}
$$

As (23), this is an exact formula, containing extensive quantities in both sides, and thus well adapted to approximations (as opposed to $Z_{\text {g.c. }}$ ). We will see below that the first term of the summation gives the grand potential f of an ideal, (degenerate), gas, which we shall note $\Xi_{\text {ideal }}$. The generalized Beth Uhlenbeck formula is contained in the second and the third term in the summation, which we shall note $\Xi_{\text {direct }}^{1}$ and $\Xi_{\text {exch. }}^{1}$; if, moreover, in each of these two classes, one limits the summation of (24) to its first term (lowest order in $U_{1}$ ), one recovers the usual formula, valid only for weakly degenerate gases.

\section{$5 \quad$ Dilute degenerate systems}

\subsection{Ideal gas}

We first check that the first class of diagrams reconstructs the grand potential (multiplied by $-\beta$ ) of the ideal gas. This class, symbolized in figure 3 -a, corresponds to the summation of the contribution of pure exchange cycles containing only $U_{1}$ 's, summed over any length $k$ ranging from one to infinity. We know from (18) the counting factor, so that we just need to calculate the numerical contribution $\Gamma_{k}$ of every cycle. The result, proved below, is simple:

$$
\Gamma_{k}=\eta^{k+1} \operatorname{Tr}\left\{\left[U_{1}\right]^{k}\right\}
$$

where:

$$
\eta=\left\{\begin{aligned}
1 & \text { for bosons } \\
-1 & \text { for fermions }
\end{aligned}\right\}
$$

This is again a consequence of the fact that the trace over the $N$ particles contained in the diagram is taken in a space that is simply the tensor product of $k$ single particle spaces of state. Because the numbering of the particles does not affect the value of $\Gamma_{k}$ (it just changes the names of dummy variables), we can for convenience renumber the relevant particles from 1 to $k$. The effect of $C_{k}$ is then to move particle 1 into the place initially occupied by particle 2 , particle 2 into the place occupied by particle 3, and so on until one comes back to the place of particle 1. Introducing a complete set of states $\left\{\mid \varphi_{n}>\right\}$ in the one particle space

\footnotetext{
${ }^{9}$ More precisely, the logarithms of partition functions give the value of the grand potential multiplied by $-\beta$.
} 
of statesty, one can then write:

$$
\begin{aligned}
\Gamma_{k}= & \eta^{k+1} \sum_{n_{1}, n_{2}, \ldots n_{k}}<1: \varphi_{n_{1}}\left|U_{1}(1)\right| 1: \varphi_{n_{2}}><2: \varphi_{n_{2}}\left|U_{1}(2)\right| 2: \varphi_{n_{3}}>\times \\
& \times \ldots \times<k: \varphi_{n_{k}}\left|U_{1}(k)\right| k: \varphi_{n_{1}}>=\eta^{k+1} \operatorname{Tr}\left\{\left[U_{1}\right]^{k}\right\}
\end{aligned}
$$

(the factor $\eta^{k+1}$ is equal to the parity of the cycle which enters in the definition of $A$ for fermions). We now have to make the summation:

$$
\Xi_{i d e a l}=\sum_{k=1}^{\infty} \frac{1}{k} e^{\beta \mu k} \Gamma_{k}=\sum_{k=1}^{\infty} \frac{1}{k} e^{\beta \mu k} \eta^{k+1} \operatorname{Tr}\left\{\left[U_{1}\right]^{k}\right\}
$$

which contains a well known series:

$$
x+\eta \frac{x^{2}}{2}+\frac{x^{3}}{3}+\eta \frac{x^{4}}{4}+\ldots=-\eta \log [1-\eta x]
$$

We therefore get for the grand potential (multiplied by $-\beta$ ) of the ideal gas:

$$
\Xi_{\text {ideal }}=-\eta \operatorname{Tr}\left\{\log \left[1-\eta e^{\beta \mu} U_{1}\right]\right\}
$$

This is the classical result. For instance, we can assume that $H_{0}$, the one-particle hamiltonian, is equal to $P^{2} / 2 m$ (kinetic energy of a particle in a box); by replacing in (31) the trace by a sum over $d^{3} k$, and $U_{1}$ by its diagonal element $e^{-\beta \hbar^{2} k^{2} / 2 m}$, one immediately recovers usual formulas that are found in most textbooks on statistical mechanics. Indeed, the method that we have used is more indirect than the traditional method, but it gives a physical interpretation to the term in $\left[U_{1}\right]^{k}$ that is obtained by expanding the logarithmic function of (31): it corresponds to the contribution of all possible cyclic exchanges of $k$ particles in the system.

\subsection{Second virial correction}

What happens now if we add the two following terms in (25), which contain one single $U_{2}$ and no Ursell operator of higher order ? Let us start with the first class of diagrams, shown in fig. 3-b where the two particles in the $U_{2}$ operator belong to two different exchange cycles, and which we will call direct diagrams. In the first diagram of this class, the two cycles are of length $k=1$ (identities) and only two particles, unaffected by exchange, are involved; this simply introduces the contribution:

$$
\Gamma_{11}=\operatorname{Tr}_{1,2}\left\{U_{2}(1,2)\right\}
$$

\footnotetext{
${ }^{10}$ If the particles have internal states, the index $n$ symbolizes at the same time the orbital quantum numbers as well as those characterizing the internal state. For instance, it the particles have spin $I$, a summation written as $\sum_{n}$ contains in fact two summations, one over orbital quantum numbers, and a second over $(2 I+1)$ spin states.
} 
The next diagram in this class corresponds to three clustered particles, two contained in the same $U_{2}$ and two in one permutation operator $C_{2}$. The numerical value of this second diagram is:

$$
\begin{aligned}
\Gamma_{2,1} & =\eta \operatorname{Tr}_{1,2,3}\left\{U_{2}(1,2) U_{1}(3) C_{2}(1,3) C_{1}(2)\right\} \\
& =\eta \operatorname{Tr}_{1,2,3}\left\{U_{2}(1,2) U_{1}(3) P_{\text {ex. }}(1,3)\right\}
\end{aligned}
$$

or:

$$
\begin{aligned}
\Gamma_{2,1} & =\eta \sum_{n_{1}, n_{2}, n_{3}} \\
& <1: \varphi_{n_{1}}\left|<2: \varphi_{n_{2}}\right| U_{2}(1,2)\left|1: \varphi_{n_{3}}>\right| 2: \varphi_{n_{2}}><\varphi_{n_{3}}\left|U_{1}\right| \varphi_{n_{1}}>
\end{aligned}
$$

which provides the following reduced value:

$$
\Gamma_{2,1}=\eta \operatorname{Tr}_{1,2}\left\{U_{2}(1,2) U_{1}(1)\right\}
$$

Similarly, one would calculate a contribution $\Gamma_{1,2}$ arising from the exchange of particles 2 and 3, and obtained by replacing in (35) $U_{1}(1)$ by $U_{1}(2)$. More generally, when $U_{2}$ clusters together $k_{1}$ particles, belonging to the same permutation cycle of length $k_{1}$, with $k_{2}$ particles belonging to another cycle of length $k_{2}$, the calculation of the effect of each of these cycles remains very similar to that of section 5.1: now we have two particles that separately exchange with others, but the algebra of operators remains the same for each of them. We therefore get the reduced value:

$$
\Gamma_{k_{1}, k_{2}}=\eta^{k_{1}-1} \eta^{k_{2}-1} \operatorname{Tr}_{1,2}\left\{U_{2}(1,2)\left[U_{1}(1)\right]^{k_{1}-1}\left[U_{1}(2)\right]^{k_{2}-1}\right\}
$$

For this class of diagrams, according to (19) the counting factor $f$ is simply $1 / 2$. The last step is to make a summation over all possible values of $k_{1}$ and $k_{2}$ after inserting an exponential of $\beta$ times the chemical potential multiplied by the number of particles contained in the diagram:

$$
\Xi_{\text {direct }}=\frac{1}{2} \sum_{k_{1}, k_{2}} e^{\beta \mu\left(k_{1}+k_{2}\right)} \Gamma_{k_{1}, k_{2}}
$$

This operation can be done by using the relation:

$$
\sum_{k=1}^{\infty}(\eta x)^{k-1}=\frac{1}{1-\eta x}
$$

One therefore introduces fractions of the $U_{1}$ 's operators, which results in the expression:

$$
\Xi_{\text {direct }}^{1}=\frac{1}{2} \operatorname{Tr}_{1,2}\left\{U_{2}(1,2) \frac{e^{\beta \mu}}{1-\eta e^{\beta \mu} U_{1}(1)} \frac{e^{\beta \mu}}{1-\eta e^{\beta \mu} U_{1}(2)}\right\}
$$


For the second class of diagrams, exchange diagrams shown in figure 3-c, the two particles contained in $U_{2}$ are intermixed inside the same circular permutation. The first exchange diagram corresponds to the two particles contained in the same transposition:

$$
\Gamma_{1,1}^{e x}=\operatorname{Tr}_{1,2}\left\{U_{2}(1,2) C_{2}(1,2)\right\}=\operatorname{Tr}_{1,2}\left\{U_{2}(1,2) P_{\text {ex. }}\right\}
$$

The second will contain three particles:

$$
\Gamma_{1,2}^{e x .}=\operatorname{Tr}_{1,2,3}\left\{U_{2}(1,2) U_{1}(3) C_{3}(1,2,3)\right\}
$$

which is equal to:

$$
\begin{aligned}
\Gamma_{1,2}^{\text {ex. }}= & \sum_{\substack{n_{1}, n_{2}, n_{3}\\
}}<1: \varphi_{n_{1}}\left|<2: \varphi_{n_{2}}\right| \\
& U_{2}(1,2)\left|1: \varphi_{n_{2}}>\right| 2: \varphi_{n_{3}}><\varphi_{n_{3}}\left|U_{1}\right| 1: \varphi_{n_{1}}>
\end{aligned}
$$

Now, we can use the equality:

$$
\begin{aligned}
& <1: \varphi_{n_{1}}\left|<2: \varphi_{n_{2}}\right| U_{2}(1,2)\left|1: \varphi_{n_{2}}>\right| 2: \varphi_{n_{3}}>= \\
& \quad<1: \varphi_{n_{1}}\left|<2: \varphi_{n_{2}}\right| U_{2}(1,2) P_{\text {ex. }}\left|1: \varphi_{n_{3}}>\right| 2: \varphi_{n_{2}}>
\end{aligned}
$$

which allows us to get the same summation over indices as in equation (34) and to obtain the reduced value:

$$
\Gamma_{1,2}^{\text {ex. }}=\operatorname{Tr}_{1,2}\left\{U_{2}(1,2) P_{\text {ex. }} U_{1}(1)\right\}
$$

Another, very similar, term occurs if the circular permutation $C_{3}(1,2,3)$ of (41) is replaced by $C_{3}(1,3,2)$; the calculation can easily be repeated and provides the result:

$$
\Gamma_{2,1}^{\mathrm{ex}}=\operatorname{Tr}_{1,2}\left\{U_{2}(1,2) P_{\text {ex. }} U_{1}(2)\right\}
$$

From the preceding equations it is not difficult to see that the generic term of this second class of diagrams is obtained from (36) by a simple replacement of $U_{2}$ by the product $U_{2} P_{\text {ex. }}$. Inserting a $P_{\text {ex }}$. into (39) therefore provides $\Xi_{\text {exch. }}^{1}$. Finally, the value of the grand potential (multiplied by $-\beta$ ), to first order in $U_{2}$, is given by:

$$
\log Z_{\text {g.c. }}=\Xi_{\text {ideal }}+\operatorname{Tr}_{1,2}\left\{e^{2 \beta \mu} U_{2}(1,2) \frac{\left[1+\eta P_{\text {ex. }}\right]}{2} \frac{1}{1-\eta e^{\beta \mu} U_{1}(1)} \frac{1}{1-\eta e^{\beta \mu} U_{1}(2)}\right\}
$$

This result is valid within an approximation which is basically a second virial treatment of the interactions, while it contains all statistical corrections. The formula remains therefore valid if the degree of degeneracy of the gas is significant. Nevertheless, as pointed out for instance in section 2.1 of ref. [12] and in ref. [13], virial series (even summed to infinity) are no longer appropriate beyond values 
where the density exceeds that of a phase transition; this is because singularities in the thermodynamic quantities occur at a transition (in the limit of infinite systems). Therefore, for bosons, the validity of (46) is limited to non condensed systems. The discussion of what happens when a Bose Einstein condensation takes place will be given in [11].

Equation (46) is a generalization of the Beth Uhlenbeck formula, and reduces to it if the two denominators containing $U_{1}$ 's are replaced by one, an operation which is valid in the limit of low densities where $e^{\beta \mu}$ is small. To see the equivalence between the low density limit of relation (46) with the usual value of the second virial correction, we start from the definition of the $W_{k}$ functions given in equation (14.35) of reference [B], which for $k=2$ becomes $\square$ :

$$
W_{2}\left(\mathbf{r}_{1}, \mathbf{r}_{2}\right)=2\left(\lambda_{T}\right)^{6} \sum_{n_{s}}\left|\Psi_{n_{s}}\left(\mathbf{r}_{1}, \mathbf{r}_{2}\right)\right|^{2} e^{-\beta E_{n_{s}}}
$$

where the functions $\Psi_{n_{s}}\left(\mathbf{r}_{1}, \mathbf{r}_{2}\right)$ are a complete set of stationary states for the system of identical particles (they are properly symmetrized); the thermal wavelength is defined by:

$$
\lambda_{T}=\frac{h}{\sqrt{2 \pi m k_{B} T}}
$$

Relation (47) can be transformed into:

$$
W_{2}\left(\mathbf{r}_{1}, \mathbf{r}_{2}\right)=2\left(\lambda_{T}\right)^{6}<1: \mathbf{r}_{1}, 2: \mathbf{r}_{2}\left|\sum_{n_{s}}\right| \Psi_{n_{s}}><\Psi_{n_{s}}\left|e^{-\beta H}\right| 1: \mathbf{r}_{1}, 2: \mathbf{r}_{2}>
$$

Inside this equation appears a closure summation over the symmetrized states of the system so that:

$$
W_{2}\left(\mathbf{r}_{1}, \mathbf{r}_{2}\right)=2\left(\lambda_{T}\right)^{6}<1: \mathbf{r}_{1}, 2: \mathbf{r}_{2}\left|S e^{-\beta H}\right| 1: \mathbf{r}_{1}, 2: \mathbf{r}_{2}>
$$

(for fermions, $S$ is replaced by $A$ ). From this function ref. [8 defines the Ursell function $U_{2}^{H}\left(\mathbf{r}_{1}, \mathbf{r}_{2}\right)$ by:

$$
U_{2}^{H}\left(\mathbf{r}_{1}, \mathbf{r}_{2}\right)=W_{2}\left(\mathbf{r}_{1}, \mathbf{r}_{2}\right)-W_{1}\left(\mathbf{r}_{1}\right) W_{1}\left(\mathbf{r}_{2}\right)=\Delta W_{2}\left(\mathbf{r}_{1}, \mathbf{r}_{2}\right)+U_{2}^{\text {ideal gas }}\left(\mathbf{r}_{1}, \mathbf{r}_{2}\right)
$$

where $\Delta W_{2}\left(\mathbf{r}_{1}, \mathbf{r}_{2}\right)$ is the difference between the values of $W_{2}\left(\mathbf{r}_{1}, \mathbf{r}_{2}\right)$ with and without interaction potential, and $U_{2}^{\text {ideal gas }}\left(\mathbf{r}_{1}, \mathbf{r}_{2}\right)$ the value of the Ursell function for a system of two free particles. Equation (14.49) [2] of [8] shows that the second virial coefficient in the expansion of the grand potential is half of the integral of $U_{2}^{H}\left(\mathbf{r}_{1}, \mathbf{r}_{2}\right)$ over the variables $\mathbf{r}_{1}$ and $\mathbf{r}_{2}$, multiplied by $\left(\lambda_{T}\right)^{-3}$ and the inverse of the volume. But the contribution of $U_{2}^{\text {ideal gas }}\left(\mathbf{r}_{1}, \mathbf{r}_{2}\right)$ is automatically contained in $\log Z_{\text {g.c. }}$, so that we can concentrate on $\Delta W_{2}\left(\mathbf{r}_{1}, \mathbf{r}_{2}\right)$ only. Because the summation

\footnotetext{
${ }^{11}$ We assume for simplicity that the particles have no internal state (or, if they do, that they are all in the same internal state).

${ }^{12}$ In the second edition, this equation is numbered (10.49).
} 
over $\mathbf{r}_{1}$ and $\mathbf{r}_{2}$ can be written as a trace in a space which is the tensor product of two one particle state spaces, and because:

$$
S, A=\frac{1}{2}\left[1 \pm P_{\text {ex. }}\right]
$$

we get the result:

$$
\int d^{3} r_{1} d^{3} r_{2} \Delta W_{2}^{V}\left(\mathbf{r}_{1}, \mathbf{r}_{2}\right)=2\left(\lambda_{T}\right)^{6} \operatorname{Tr}_{1,2}\left\{U_{2}(1,2) \frac{\left[1+\eta P_{\text {ex. }}\right]}{2}\right\}
$$

where $U_{2}(1,2)$ is the difference between the exponentials of the interacting particle hamiltonian minus that of free particles, which is precisely our definition (3). We therefore recover our result (46), provided the denominators $1-\eta e^{\beta \mu} U_{1}$ inside the trace are replaced by one.

\subsection{Discussion; reduced density operators}

The only difference between equation (46) and the Beth Uhlenbeck formula arises from the presence of the two fractions inside the trace. Since:

$$
\frac{1}{1-\eta e^{\beta \mu} U_{1}}=1+\eta \frac{e^{\beta \mu} U_{1}}{1+\eta e^{\beta \mu} U_{1}}
$$

they are actually nothing but operatorial forms of the usual Fermi or Bose factors $(1+\eta f)$ that appear, for instance, in the collision term of the Uehling Uhlenbeck or Landau kinetic equation (with the usual notation $f$ for the distribution function). In the present case, nevertheless, because $U_{2}$ and $U_{1}$ do not commute in general, the operatorial character of the fractions is relevant. For free (but mutually interacting) particles in a box, the eigenvectors of $U_{1}$ are plane waves $[3$, while those of $U_{2}$ are different since they involve correlations between the particles.

If the trace in the right hand side of (46) is calculated in the basis of plane waves, the correction is expressed as an integral containing the diagonal elements of $U_{2}$ between such plane waves. The simplest situation occurs when all these diagonal elements have the same sign. Then, for bosons, because the eigenvalues of the fractions are larger than 1, the effect of degeneracy is always to enhance the effects of interactions; this is physically satisfying since the Bose Einstein statistics tends to favor situations where particles are close. In particular, if the potential is attractive and if there are two body bound states (molecules), their weight will be increased with respect to what is would be in the usual Beth Uhlenbeck formula. If, on the other hand, the diagonal elements of $U_{2}$ between plane waves change sign when the relative momentum of the two particles changes, which may

\footnotetext{
${ }^{13}$ If the particles are subject to the effect of an external potential (atoms in a trap for instance), the eigenvectors of $U_{1}$ are not simple plane waves, but the essence of our analysis remains valid.
} 
happen if the potential has attractive as well as repulsive parts, more complicated cancellation effects may take place in both the usual Beth Uhlenbeck formula and its generalization, so that no general prediction on the sign of the effect of degeneracy is possible (except for the contribution of bound states which remains enhanced as above).

For fermions, the eigenvalues of the fractions are between 0 and 1 so that the effect of statistics are just the opposite of what they are for bosons: they tend to reduce the effects of the interactions, except if mutual cancellation effects take place. Moreover, if the system is strongly degenerate, the effect of the product of the two fractions is to cancel the contribution of all matrix elements corresponding to particles inside the Fermi sphere, leaving only interactions between particles near the surface (or outside) of the Fermi sphere. This applies to bound molecules, which introduce a contribution containing the scalar product of the bound state wave function by all plane waves outside of the Fermi sphere, exactly as in the Cooper problem.

The applicability of (46) is not limited to the equation of state of the quantum system, it can also be applied to the evaluation of partial density operators, for instance the one particle density operator. If we set ${ }^{\text {T4: }}$

$$
U_{l}(1,2, . . l)=\left[U_{1}(1) \times U_{1}(2) \times \ldots \times U_{1}(l)\right] \bar{U}(1,2, . . l)
$$

we can choose $U_{1}$ and the $\bar{U}_{l}$ 's, instead of the $U_{l}$ 's, to characterize the correlations between the particles; by definition $\bar{U}_{1}$ is equal to one. Assume now that we keep all $\bar{U}_{l}$ 's constant and vary $U_{1}$ according to:

$$
d U_{1}=d \varepsilon|\varphi><\varphi| U_{1}
$$

What is then the variation of $Z$ ? Inserting this relation into (55), then into (4) and finally into (11), we get:

$$
d Z_{N}=d \epsilon \operatorname{Tr}_{1,2,3, \ldots N}\left\{\begin{array}{l}
S \\
A
\end{array} K_{N} \sum_{i=1}^{i=N}|i: \varphi\rangle\langle i: \varphi|\right\}
$$

This expression is nothing but the product of $Z_{N}$ by the average value of the one particle operator $\sum_{i=1}^{i=N}|i: \varphi\rangle\langle i: \varphi|$. Therefore, in terms of the one particle density operator $\rho_{I}$, we obtain:

$$
\frac{d}{d \epsilon} \log Z_{N}=N \operatorname{Tr}\left\{|\varphi\rangle\langle\varphi| \rho_{I}\right\}=N\left\langle\varphi\left|\rho_{I}\right| \varphi\right\rangle
$$

(the derivative with respect to $\epsilon$ is meant at the value $\epsilon=0$ ). We can therefore obtain reduced density matrices from this kind of operatorial variations. If the

\footnotetext{
${ }^{14}$ If one wishes to introduce hermitian operators only, one can symmetrize (55) by putting square roots of $U_{1}$ 's on both sides of $\bar{U}$.

${ }^{15}$ Here we take the convention where the operator $\rho_{I}$ is normed to the number $N$ of particles (instead of one).
} 
grand canonical ensemble is used, the many-particle density operator is defined by:

$$
\rho_{g c}=\left[Z_{g c}\right]^{-1} \sum_{N} e^{\beta \mu N}\left\{\begin{array}{l}
S \\
K_{N}
\end{array}\right.
$$

and we find the following relation involving the one particle density operator $\rho_{I}^{g c}$ :

$$
\frac{d}{d \epsilon} \log Z_{g c}=\operatorname{Tr}\left\{\rho_{I}^{g c}|\varphi\rangle\langle\varphi|\right\}=\left\langle\varphi\left|\rho_{I}^{g c}\right| \varphi\right\rangle
$$

This formula shows that $\rho_{I}^{g c}$ is the "operatorial derivative" of $Z_{g c}$ (we have only considered diagonal variations of $U_{1}$ but, since the basis of states $|\varphi\rangle$ is arbitrary, a generalization to off-diagonal variations is easy).

For the ideal gas, all $\bar{U}_{l}$ are zero if $l \geq 2$. We can then simply use (31) and take a derivative with respect to $\varepsilon$ by choosing in succession for $|\varphi\rangle$ all eigenvectors of $\rho_{F}$. In this way we obtain:

$$
\rho_{I}^{g c}=\frac{e^{\beta \mu} U_{1}}{1-\eta e^{\beta \mu} U_{1}}
$$

which is a well known result. When interactions are added, one should replace (31) by (46) with the substitution (55) for $U_{2}$. One then gets a modified expression of $\rho_{I}$ where contributions arise from the derivatives of the denominators in (46) as well as that of the numerator (through the variation of $U_{2}$ ), that we do not write here for brevity. This shows how the method is adaptable to the evaluation of microscopic quantities; if one is interested in microscopic correlations, two body density operators can be obtained by a similar method. This is a specificity of our technique, as compared for instance to that based on the pseudopotential; the latter is built in order to reconstruct the eigenvalues of the hamiltonian, and hence the partition function, but not the wave functions, in particular certainly not at short relative distances.

A final remark is related to the convergence of the power series that we have summed into fractions of the $U_{1}$ operators (the remark applies for fermions only). As noted by Kahn and Uhlenbeck [6], when the chemical potential of a system of fermions becomes positive, the virial series diverge, and the equation of state is obtained by a continuation of a an analytic function. Here we observe the same

phenomenon: the series in $\left[e^{\beta \mu} U_{1}\right]^{k}$ that we have summed over the size $k$ of the exchange cycles becomes divergent when $\mu>0$; nevertheless the sum remains a regular function and, for fermions, (46) has no singularity.

\section{Conclusion}

The basic idea of the method of Beth and Uhlenbeck, or more generally of Mayer cluster expansion methods [16], is to reason in terms of functions which directly 
give local approximations of the thermal equilibrium. Here we start from Ursell operators which give rise to various contributions in the form of U-C diagrams. This leads to equation (25) which provides an exact expression of the grand potential of the system, and expresses it as sum of various terms arising from $\Xi$-diagrams, already containing a sum over all possible sizes of intermediate exchange cycles. Each term is obtained as an integral (a trace) over a finite number of variables. The general expression is valid for dilute or dense systems as well, such as liquids or even solids, but for gases it becomes simpler because it can be truncated more abruptly. Indeed, the generalization of the Beth Uhlenbeck formula is obtained by limiting the summation to the first three terms only (the first corresponding to the ideal gas).

In its spirit, the method that we have used is close to the "binary collision approximation of Lee and Yang [14] 15]"; they also introduce the Ursell functions of an auxiliary system obeying Boltzmann statistics, and they establish relations between the Ursell functions of the two systems (see also ref. [17 for a discussion of this type of method). But these relations acquire a rapidly increasing complexity with the order of the Ursell functions, and since they do not use Ursell operators, it becomes necessary at some point to make approximations (integrations over the inverse temperature $\beta$ ). In our method, we introduce operators which generalize Ursell functions, and we make no attempt to evaluate the Ursell functions of the system of identical particles. Rather, we calculate the complete contribution of each initial (unsymmetrized) Ursell operator to the symmetrized partition function; this explains why the final results are markedly different.

Another point of comparison is the more recent calculation by Nozières and Schmitt Rink [18], who give a calculation of the thermodynamic potential for a system of fermions at low densities. In order to simplify the summation of the diagrams in their calculation, they assume that the matrix elements of the interaction potential are separable into a product of functions; moreover, for brevity, they explicitly include only one phase shift (s wave approximation) so that their result is less general than (46). Of course this does not mean that their method can not be generalized to fully recover (46), but we have not examined the question. In the same vein, a more detailed comparison between (46) with the results of Galitskii [5] for fermions and Belyaev [19] for bosons, valid a zero temperature, would be useful.

\section{ACKNOWLEDGMENTS}

Part of this work was done during a pleasant and interesting visit in Leiden at the Kamerling Onnes Laboratory; the author is very grateful to his host Prof. Giorgio Frossati, as well to his co-visitor Prof. Sandro Stringari, for discussions and intellectual stimulation. Several useful and friendly discussions with Philippe Nozières are also acknowledged. The text of this article owes a lot to Prof. W. Mullin and to Peter Grüter, who both suggested corrections and improvements. 


\section{APPENDIX I : GENERAL RULES FOR CONSTRUCTING DIAGRAMS}

Our convention for building the diagram associated to any particular term in the double summation (9) is the following:

(i) we start from the $U_{l}$, or the $U_{l}$ 's, that are of highest order $l=l_{M}$ in this particular term, and identify the particle it (or they) contain(s) that has the lowest index number $n_{\min }$; this particle is considered as the "base particle", and belongs to the "base cycle" from which all the rest of diagram will be drawn. By convention, the base particle is shown first (left position) and the base cycle is that at the lowest position in the figure; for instance, in figures 1-b and 1-d, this base is a three particle cycle.

(ii) to continue in the construction of the diagram, we add a second generation of cycles. We first use the Ursell operator $U_{l_{M}}$ that contains the base particle and add the other permutation cycles which include the other particles in the same $U_{l_{M}}$; this is done in the order of increasing values for the index number of the particles contained in this $U_{l_{M}}$, so that the order of the new cycles is clearly defined. This also defines, for each of them, a "secondary base particle" that is put first in the diagram

(iii) we continue the addition of this second generation of branches in the diagram by moving along the base cycle and skipping all numbered particles that are in $U_{1}$ 's, until we reach one which belongs to an $U_{l}$ with $l \geq 2$; we then add additional cycles containing the other particles inside this $U_{l}$. We use the same rule an in (ii) and we define "secondary bases" for the new cycles, so that their representation is also uniquely fixed. Going along all the base cycle in this way completes the first generation of additional cycles.

(iv) Then we build in the same way the second generation, by starting in succession from all of cycles of the first generation in the order in which they were added, etc. until, eventually, the complete diagram is obtained.

With these conventions, all cycles are individually identified, so that it makes sense to vary their lengths independently to generate all terms of (9); this will be useful below for the calculations which lead from the U-C to the $\Xi$-diagrams. Figure 2 shows examples of diagrams; Appendix II gives more details on the conventions used in their representation (in particular, it is convenient to assume that, inside the trace, the series of Ursell operators are put before the series of cycles).

Now suppose that we reverse the question: starting from a given diagram, how do we identify its occurrence in the double sum? what is its weight in the summation that gives $\log Z$ ? Assume that we throw randomly $n_{\text {diag. }}$. numbered particles into all available locations. With the precise rules that we have chosen

\footnotetext{
${ }^{16}$ If the connection is multiple, that is if the additional cycle contains several particles of the same base Ursell operator $U_{l_{m}}$, the secondary base particle is that of lowest index number; all the other particles are then automatically located in the diagram by their order in the exchange cycle, and do not play a special role at this stage.
} 
above, it is clear that double counting problems are avoided, but also that not all of these random configurations obtained are allowed. What is the proportion $f_{\text {diag. }}$ of the configurations that are compatible with our conventions? Let us note $p_{l_{M}}$ the total number of operators $U_{l_{M}}$ (those of largest order $l_{M}$ ) appearing in this particular diagram. The reasoning is as follows:

(i) first there is a probability $\left(p_{l_{M}} \times l_{M}\right)^{-1}$ that the right base particle will be obtained

(ii) second, if $l_{M} \geq 3$, in the construction of successive generations of cycles, either more than one cycle is added from the connections of the same $U_{l}$, or there is a multiple connection towards the same cycle (or both). Figure 1-e gives one example of such a multiple connection. In all these cases, additional factors $(g)^{-1}$ are introduced which account for the correct ordering of numbering of secondary base particles.

Thus we obtain:

$$
f_{\text {diag. }}=\left[p_{l_{M}} \times l_{M} \times g\right]^{-1}
$$

(this formula is also valid if $l_{M}=1$, in which case $p_{l_{M}}$ is nothing but the size $k$ of the linear exchange cycle). For the generalization of the Beth Uhlenbeck formula, case (ii) never happens and the factors $g$ 's do not play any role; they would nevertheless enter the calculations in general, for instance in the discussion of Bose Einstein condensation of groups of three particles in [11].

\section{APPENDIX II : TWO EXAMPLES}

The diagram shown in figure 2-a corresponds by definition to the following trace:

$$
\operatorname{Tr}_{1,2,3,4,5}\left\{U_{2}(1,2) U_{2}(3,5) U_{1}(4) C_{1}(1) C_{3}(2,3,4) C_{1}(5)\right\}
$$

Our convention is that the Ursell operators are always put before the cycles; the notation $C_{3}(2,3,4)$ refers to a cycle where the particle numbered 2 replaces that numbered 3, that numbered 3 replaces that numbered 4 , and that numbered 4 that numbered 2 (the $C_{1}$ 's do not produce any change in the positions of the particles). Equation (63) gives what we call the "explicit value" of this particular diagram, but it can also be simplified into a "reduced value". This can be done by inserting into (63) closure relationships and using summations to introduce products of operators whenever possible. In this case this leads to the expression:

$$
\operatorname{Tr}_{1,2,5}\left\{U_{2}(1,2) U_{2}(2,5) U_{1}(2)\right\}
$$

In the explicit value, any numbered particle appears once and only once in every Ursell operator and every cycle; in the reduced form this is not necessarily the case.

In a similar way the diagram shown in figure 2-b is defined by the explicit expression:

$$
\operatorname{Tr}_{1,2,3,4,5,6}\left\{U_{2}(1,2) U_{1}(3) U_{2}(4,5) U_{1}(6) C_{1}(1) C_{3}(2,3,4) \eta C_{2}(5,6)\right\}
$$


while its reduced value is:

$$
\eta \operatorname{Tr}_{1,2,5}\left\{U_{2}(1,2) U_{1}(2) U_{2}(2,5) U_{1}(5)\right\}
$$

\section{FIGURE CAPTIONS}

Fig. 1: Examples of U-C diagrams. For an ideal gas, only linear U-C diagrams containing chains of one particle Ursell operators $U_{1}$ occur, as shown in (a); a summation of the contribution of these diagrams over the length of the chain gives the grand potential (multiplied by $-\beta$ ). The generalization of the Beth Uhlenbeck formula arises for the diagrams shown in (b) and (c) containing one single two body Ursell operator $U_{2}$; for each of them, a summation over the lengths of the $U_{1}$ chains is also necessary. Figure (d) shows an example of a diagram containing a three body $U_{3}$ operator and three $U_{1}$ chains, with a counting factor $g=2$. Figure (e) shows an example of multiple connection through an $U_{3}$ operator, which also introduces a factor $g=2$ in the weight of the diagram. For more details on the definition and counting of the diagrams, see Appendix I.

Fig. 2: Other examples of more complex U-C diagrams. As in figure 1, horizontal lines correspond to exchange cycles containing $U_{1}$ operators; in addition, vertical double lines symbolize $U_{2}$ operators (triple lines would be used for $U_{3}$, etc.). Formula (19) gives the weight of these diagrams; a more explicit calculation of these two terms is given in Appendix II.

Fig. 3: $\Xi$ diagrams introduced by the summation of the diagrams of figure 1 over the lengths of the $U_{1}$ chains, according to formula (24). The dashed lines, which symbolize these summations, can be replaced by intermediate operators given by fractions $1 /\left[1-\eta z U_{1}\right]$, where $z=\exp \beta \mu$; every operator $U_{l}$, with $l \geq 2$,

remains explicit and, moreover, introduces a factor $z^{l}$; finally, the weights $f_{\text {diag }}$. must also be inserted in the value of the $\Xi$ diagram.

\section{BIBLIOGRAPHY}

\section{References}

[1] E. Beth and G.E Uhlenbeck, Physica 3, 729 (1936) and 4, 915 (1937).

[2] K. Huang, Statistical Mechanics, § 14.3, Wiley (1963); second edition (1987) $\S 10.3$.

[3] K. Huang, loc. cit. $\S \S 13.4$ and 13.5; chap. 19. 
[4] E.M. Lifshitz and L.P. Pitaevskii, Landau and Lifshitz course of theoretical physics vol. 9, Statistical Physics, Part 2, §6 and 25.

[5] V.M. Galitskii, Soviet Phys. JETP 34, 104 (1958).

[6] B. Kahn and G.E. Uhlenbeck, Physica 5, 399 (1938).

[7] H.D. Ursell, Proc. Camb. Phil. Soc. 23, 685 (1927).

[8] K. Huang, loc. cit. chap 14; second edition (1987), chap. 10.

[9] R.K. Pathria, Statistical Mechanics, Pergamon Press 1972, §9.6

[10] F. Laloë, contribution to "Bose Einstein Condensation" ed. by A. Griffin, D.W. Snoke and S. Stringari, Cambridge University Press, 1993.

[11] P. Grüter and F. Laloë, to be submitted to the Journal de Physique.

[12] K Huang, "Imperfect Bose gas", in Studies in Statistical Mechanics vol. II, editors J. de Boer and G.E. Uhlenbeck, North Holland 1964.

[13] C.N Yang and T.D. Lee, Phys. Rev. 87, 404 (1952).

[14] T.D. Lee and C.N. Yang, Phys. Rev. 113, 1165 (1959); 116, 25 (1959).

[15] R.K. Pathria, loc. cit. $§ 9.7$

[16] J.E. Mayer, Journ. Chem. Phys. 5, 67 (1937); J.E. Mayer and P.G. Ackermann, ibid., page 74; J.E. Mayer and S.F. Harrisson ibid. 6, 87 (1938); S.F. Harrisson and J.E. Mayer, ibid. page 101.

[17] F. Mohling, Phys. Rev. 135, A831 (1964).

[18] P. Nozières and S. Schmitt Rink, J. Low Temp. Phys 59, 195 (1984).

[19] S.T. Belyaev, Sov. Phys. JETP 34, 189 (1958). 